\title{
Softwares de Geometria Interativa para Deficientes Físicos e Intelectuais: Um Mapeamento Sistemático
}

\section{Interactive Geometry software for physical and intellectual disability students: A Systematic Mapping}

\author{
${ }^{1}$ João Henrique Sass de Souza (j_henrique95@outlook.com) \\ ${ }^{1}$ Igor Roberto Guilherme (igor.guilherme09@ hotmail.com) \\ ${ }^{2}$ Simone de Sousa Borges (sborges@icmc.usp.br) \\ ${ }^{1}$ Ramílio Ramalho Reis Filho (ramilio.ramalho@ hotmail.com) \\ ${ }^{3}$ Helena Macedo Reis (helenareis@utfpr.edu.br) \\ ${ }^{1}$ Faculdade de Tecnologia de Taquaritinga \\ Taquaritinga, Brasil \\ ${ }^{2}$ Instituto de Ciências Matemáticas e de Computação - ICMC \\ Universidade de São Paulo - USP \\ São Carlos, Brasil \\ ${ }^{3}$ Universidade Tecnológica Federal do Paraná \\ Dois Vizinhos, Brasil
}

\begin{abstract}
The use of Interactive Geometry software can facilitate learning and improve the quality of education for people with physical and intellectual limitations. Nevertheless, approaches to the development of these software are poorly investigated. To provide an overview of the results already obtained in this area, a systematic mapping was conducted in order to analyze software or additional devices have been used. In total, 451 studies were analyzed in more than 10 years of research in the area of interactive geometry. Among these, 45 studies were related to the development of interfaces and only 24 of them satisfied the inclusion and exclusion criteria defined in this work. As a result, we verified that most studies have focused software for visually and hearing impaired persons, being mostly for children. We also identified that there are many research opportunities in developing software for physical and intellectual disabled for geometry learning
\end{abstract}

Keywords: Interactive geometry, physical limitation, intellectual limitation, assistive technology.

Resumo. $O$ uso de softwares de Geometria Interativa pode facilitar o aprendizado e melhorar a qualidade de ensino para pessoas que possuem limitações físicas e intelectuais. Apesar disto, as abordagens sobre o desenvolvimento destes softwares são pouco investigadas. Para oferecer uma visão geral dos resultados já obtidos nesta área, um mapeamento sistemático foi conduzido com o objetivo de analisar quais softwares atendem este público e em quais dispositivos adicionais estes softwares utilizam. No total foram 451 estudos analisados em mais de 10 anos de pesquisa na área de geometria interativa. Dentre estes estudos, 24 deles estavam relacionados com softwares 
de geometria interativa e apenas 20 deles satisfizeram os critérios de inclusão e exclusão definidos neste trabalho. Como resultado, verificou-se que maioria dos estudos apresentam softwares voltados para deficientes visuais e auditivos, sendo sua maioria para crianças. Este resultado demonstra que existem diversas oportunidades de pesquisa no desenvolvimento de softwares para deficientes físicos e intelectuais para aprendizagem de geometria

Palavras-chave: Geometria interativa, limitação física, limitação intelectual,
tecnologia assitiva.

\section{Introdução}

Segundo um estudo feito pela Organização Mundial da Saúde (OMS) em 2016, em torno de 650 milhões de pessoas possuem algum tipo de deficiência no mundo. Este estudo evidenciou que cerca de 1 milhão destas pessoas tem estão em fase escolar, mas estão fora das escolas brasileiras, necessitando de tecnologias para apoiar a aprendizagem, segundo o Ministério da Educação (MEC). Dentre as tecnologias necessárias para este público, os sistemas para o ensino da geometria (conhecidos sistemas de Geometria Interativa - GI) são indispensáveis e precisam ser aprimoradas e popularizadas, para que todos possam usufruir dos seus benefícios.

Os sistemas de GI, também conhecida como Geometria Dinâmica (Silva et al., 2013) foram desenvolvidos visando proporcionar aos estudantes a possibilidade de criar formas geométricas em um ambiente computacional de maneira intuitiva. Estes sistemas de ensino de geometria utilizam de linguagem visual e linguagem audiovisual, tornando mais palpável para o entendimento do estudante. Além disso, permitem o estudante receber um retorno visual em tempo real a cada modificação feita, sendo uma vantagem sobre o modelo tradicional (e.g. o uso do lápis e papel) (Isotani e Brandão, 2004; Reis et al., 2012).

Apesar da importância dos sistemas de GI para a aprendizagem, alguns desses sistemas (e.g. Geogebra (Hohenwarter, 2001), Cinderella (Kortenkamp, 1999), CAR (Grothman, 1999)) não foram desenvolvidos considerando as limitações dos estudantes que possuem algum tipo de deficiência física ou intelectual. Existem poucos estudos que exploram o desenvolvimento de sistemas de SI para este público.

Dessa forma, este trabalho apresenta os resultados de um mapeamento sistemático elaborado com o propósito de investigar quais tecnologias, como softwares e dispositivos adicionais são utilizados para o ensino da geometria para pessoas deficientes. Mapeamentos sistemáticos são uma forma de categorizar e sintetizar informações existentes sobre uma determinada área de interesse, respondendo a questões de pesquisa de modo imparcial (Petersen, 2008). O restante deste artigo está organizado da seguinte forma: Seção 2 apresenta o processo do mapeamento sistemático, feito com base nos dados obtidos na análise de outros artigos sobre o presente tema. Seção 3 apresenta a classificação dos estudos que serão feitos com determinada deficiência, e Seção 4 apresenta o resultado do mapeamento feito e as respostas obtidas com o estudo. Na Seção 5 , é apresentada uma visão geral sobre o uso da tecnologia no ensino de geometria. $\mathrm{Na}$ Seção 6 é discutido possíveis ameaças para a validação do artigo, e por último, a Seção 7 apresenta a conclusão deste artigo. 


\section{O processo de mapeamento sistemático}

Este estudo se baseou na estrutura para construção de um mapeamento sistemático seguindo as diretrizes propostas por Petersen et al. (2008) e foi elaborado de acordo com cinco etapas: (i) definição das questões de pesquisa (QP), (ii) pesquisa de estudos primários relevantes, (iii) seleção de estudos primários, (iv) categorização dos artigos e (v) mapeamento e extração de informações de acordo com as questões de pesquisa levantadas previamente. Todas as etapas foram executadas sequencialmente e quantas vezes necessárias. Quando todas as etapas forem concluídas o resultado será o mapeamento sistemático.

As seguintes questões de pesquisa foram feitas para responder como os softwares de GI estão sendo utilizadas para ajudar no ensino de geometria para pessoas com deficiência e sua eficácia, as questões elaboradas foram as seguintes:

QP1: Quais são os principais tipos de deficiência investigados para softwares de GI?

$Q_{1.1}$ : Para qual faixa etária estes softwares de GI foram desenvolvidos?

$\boldsymbol{Q P}_{2}$ : Quais dispositivos adicionais que estes softwares de GI usualmente utilizam?

QP3: Quais softwares adicionais que estes softwares de GI usualmente utilizam?

Com base nestas questões elaboradas foram definidos critérios de inclusão e exclusão de artigos, para ter uma melhor filtragem e garantir que apenas artigos relevantes de sejam incluídos nos estudos. Foram determinados os seguintes critérios para inclusão:

- Artigos que investigam o uso da tecnologia para auxiliar o aprendizado de geometria para pessoas que possuam algum tipo de deficiência.

- Se houver várias versões de um mesmo artigo, apenas a mais completa será incluída no estudo.

- Se vários artigos mostram estudos similares, somente o mais recente deverá ser incluído;

E os critérios para exclusão foram os seguintes:

- Artigos que não tem alguma relação com o ensino da geometria utilizando algum tipo de tecnologia para pessoas que apresentam algum tipo de deficiência, seja um software ou um dispositivo.

- Estudos que não estejam em português ou inglês.

- Relatórios técnicos, documentos que estão disponíveis na forma de resumos ou apresentações/slides e também estudos secundários (ou seja, revisões e mapeamentos sistemáticos da literatura) serão excluídos.

A string de pesquisa utilizada nas bases de dados acadêmicas foi construída em três partes, e formatadas de acordo com a regra de cada base de dados, tanto em português como em inglês. A primeira parte da string se refere aos tipos de deficiência, a segunda parte referência o estudo da geometria e a última parte indica a relação com a tecnologia que deve ser encontrada. Nenhum filtro foi utilizado. A string em português é apresentada logo abaixo:

("deficiente físico" OR "deficiente auditivo" OR "deficiente visual" OR "deficiente mental" OR "deficiente múltiplo") AND ("Ensino de Geometria" OR "Aprendizado da 
Geometria") AND (tecnologia OR informática OR computador OR "ambiente computacional")

Foi considerado também a string em inglês com o objetivo de obter o máximo de artigos relacionados ao tema.

("physical disability" OR "mental disability" OR "intellectual disability" OR "physically handicapped" OR "mentally handicapped" OR "intellectual handicapped" OR "visually impaired" OR "hearing impaired") AND ("Dynamic Geometry" OR "Geometry Teaching” OR “Geometry Education")

A pesquisa sobre os estudos primários foi realizada no período entre 10/05/2016 e 26/05/2016. A princípio foram retornados 481 artigos somando todas as bases. Os autores deste estudo realizaram simultaneamente a leitura dos títulos, resumos e palavras-chave de todos os artigos. Cada autor gerou uma lista de estudos selecionados, que posteriormente foram analisados e discutidos a fim de definir um conjunto em comum. Ao final do conjunto de 481 artigos foi reduzido à somente 24 estudos que se relacionavam realmente ao tópico investigado. Após este passo, foi realizada novamente a leitura dos resumos, introdução e conclusão, e a aplicação dos critérios de inclusão e exclusão, o que resultou em um subconjunto de 20 estudos primários (Tabela 1). Em seguida, foi realizada a leitura integral dos estudos selecionados a fim de se proceder a categorização dos mesmos, que será discutida mais detalhadamente na próxima seção. A lista completa de artigos pode ser encontrada em: https://goo.gl/2LUBvA.

Tabela 1 - Número de estudos retornados em cada base de dados acadêmicas, número total de estudos, total de estudos candidatos à estudo primário e a seleção final de estudos primários.

\begin{tabular}{|l|c|c|c|}
\hline \multicolumn{1}{|c|}{ Biblioteca digital } & Quantidade & $\begin{array}{c}\text { Quantidade em } \\
\text { Português }\end{array}$ & $\begin{array}{c}\text { Quantidade em } \\
\text { Inglês }\end{array}$ \\
\hline ACM Digital Library & 256 & 0 & 256 \\
\hline Scopus & 25 & 0 & 25 \\
\hline Web of Knowledge & 11 & 0 & 11 \\
\hline Google Scholar & 195 & 116 & 89 \\
\hline IEEExplore & 0 & 0 & 0 \\
\hline Scielo & 3 & 3 & 0 \\
\hline Total & $\mathbf{4 8 1}$ & $\mathbf{1 1 9}$ & $\mathbf{3 7 2}$ \\
\hline Candidatos & $\mathbf{2 4}$ & $\mathbf{1 3}$ & $\mathbf{1 1}$ \\
\hline Seleção final & $\mathbf{2 0}$ & $\mathbf{9}$ & $\mathbf{1 1}$ \\
\hline
\end{tabular}

Os estudos primários foram organizados por data de publicação, foi constatado um recente crescimento no interesse de pesquisas, mais especificamente, a partir do ano de 2012. As primeiras pesquisas sugiram nos anos de 1988 e 1996, entretanto o tema não foi investigado novamente até 2004. A partir deste período, houve aumento pelo interesse em tópicos sobre acessibilidade e inclusão digital, resultando no ressurgimento das investigações do ensino da geometria com uso da tecnologia para pessoas com alguma 


\section{4}

deficiência. Apesar de uma maior frequência dos estudos nos últimos anos, ainda é pouco investigado, sendo considerada uma lacuna de pesquisa.

\section{Classificação de acordo com tipos de estudos}

Para responder à questão $\mathrm{QP}_{1}$ (Quais são os principais tipos de deficiência investigados para softwares de GI?), cada estudo primário foram classificados de acordo com duas categorias: auditiva e visual. Estas categorias foram baseadas na lei Brasileira 5.2961, qual classifica os tipos de deficiências em auditiva, física, mental, múltipla e visual. Porém, não foram encontrados estudos primários nas categorias física, mental e múltipla. Na Figura 2, percebe-se que a maioria dos estudos primários são da categoria deficiência visual, apresentando 17 estudos (85\%). Sendo assim, conclui-se que a resposta para $\mathrm{QP}_{1}$ é que a deficiência visual é a categoria onde há mais estudos para o ensino da geometria utilizando com o auxílio da tecnologia. Em contrapartida, a categoria que possui o menor número de estudos primários seria a deficiência auditiva, onde apenas três estudos (15\%) foram realizado.

Tabela 2: Número de estudos primários em cada categoria.

\begin{tabular}{|c|c|c|}
\hline Categoria & Frequência & Porcentagem (\%) \\
\hline Visual & 17 & $85 \%$ \\
\hline Auditiva & 3 & $15 \%$ \\
\hline
\end{tabular}

\subsection{Visual}

Os estudos primários, que pertencem à esta categoria, abordam o uso de diversas tecnologias para auxiliar pessoas com baixa visão ou completamente cegas no aprendizado da geometria. De acordo com Buzzi et al. (2015), a melhor forma para pessoas com deficiência visual obter informações sobre formas e objetos 3D é o toque. Uma vez que estes podem manipular estes objetos no mundo real, os estudantes podem obter uma base melhor sobre conceitos como retas, vértices e circunferências. Uma das formas dessas tecnologias auxiliar o estudante com deficiência visual é criar ou simular formas físicas em tempo real baseadas com dados sobre formas geométricas vindas de algum software. Estas tecnologias também permitem ao estudante utilizar o tato para analisar as formas em ambiente digital, sendo por meio de algum dispositivo que emita vibrações que simulem a sensação do toque ou vibrações sendo emitidas diretamente da área tocada. Em contrapartida, outra forma de transmitir informações para deficientes visuais é por meio de um retorno de informações em forma de áudio (Lirio, 2006), esta técnica consiste em um modo de passar informações sobre formas geométricas e outras funções do sistema para o estudante e também para que o estudante possa mandar informações e comandos para o sistema.

Algumas das tecnologias utilizadas para dar um retorno de informação ao estudante são impressoras 3D, luvas hápticas, canetas hápticas, joystick e trackballs (Franciosi, 1988). Os principais sistemas utilizados para auxiliar o ensino da geometria para deficientes visuais seriam: HDS (Haptic Deictic System) (Oliveira et al., 2010), MICOLE (Multimodal collaboration environment for inclusion of visually impaired children) (Moll, 2013), 2D tactile graphics editor (Ina, 1996), Geometrix (Silva et al., 2013), Dosvox (Lirio. 2006), Cityvox (Silva, 2009), GC (Geometric Constructer) (Arzarello et al., 2013), Salome (Gouy-Pailler et al., 2007).

\subsection{Auditivo}


Nesta categoria foram incluídos estudos que analisaram tecnologias que auxiliam deficientes auditivos no aprendizado da geometria. O objetivo principal destes estudos era mostrar tecnologias, como a SELP (Special Eletronic Learning Program) (Kiboss, 2012), que trazem um retorno visual para o estudante com deficiência, que necessitam de uma alternativa para as explicações faladas em sala de aula. Um exemplo de sistema seria o iGeom (Isotani et al., 2004), que é um sistema que utiliza de interações gráficas para passar ao estudante as informações necessárias. Outro exemplo é o LOGO (Martins, 2013), que consiste um sistema que por meio de animações gráficas permite o estudante com deficiência auditiva entender melhor sobre formas geométricas. Alguns estudos analisaram a eficácia do retorno visual de imagens geométricas e instruções em libras (Martins, 2013), para facilitarem o entendimento destes estudante ao conteúdo que está sendo passado.

\section{Análise}

Nesta seção foram analisados os resultados obtidos com o mapeamento sistemático. O objetivo desta seção foi obter uma visão geral sobre como a tecnologia auxilia pessoas com deficiência no aprendizado da geometria. As informações levantadas na análise dos estudos primários foram utilizadas para responder as questões de pesquisa levantas anteriormente.

A Figura 2 mostra o mapa conceitual do uso de diversas tecnologias no ensino de geometria para pessoas com deficiência visual e auditiva. Sendo divido nos três seguintes grupos: tipos de deficiência, dispositivos adicionais e softwares adicionais. (i) Tipo de deficiência: o tipo de deficiência que a pessoa possui; (ii) Dispositivo adicional: dispositivos adicionais utilizados no ensino da geometria para pessoas com deficiência e (iii) Software adicional: softwares adicionais utilizados no ensino da geometria para pessoas com deficiência.

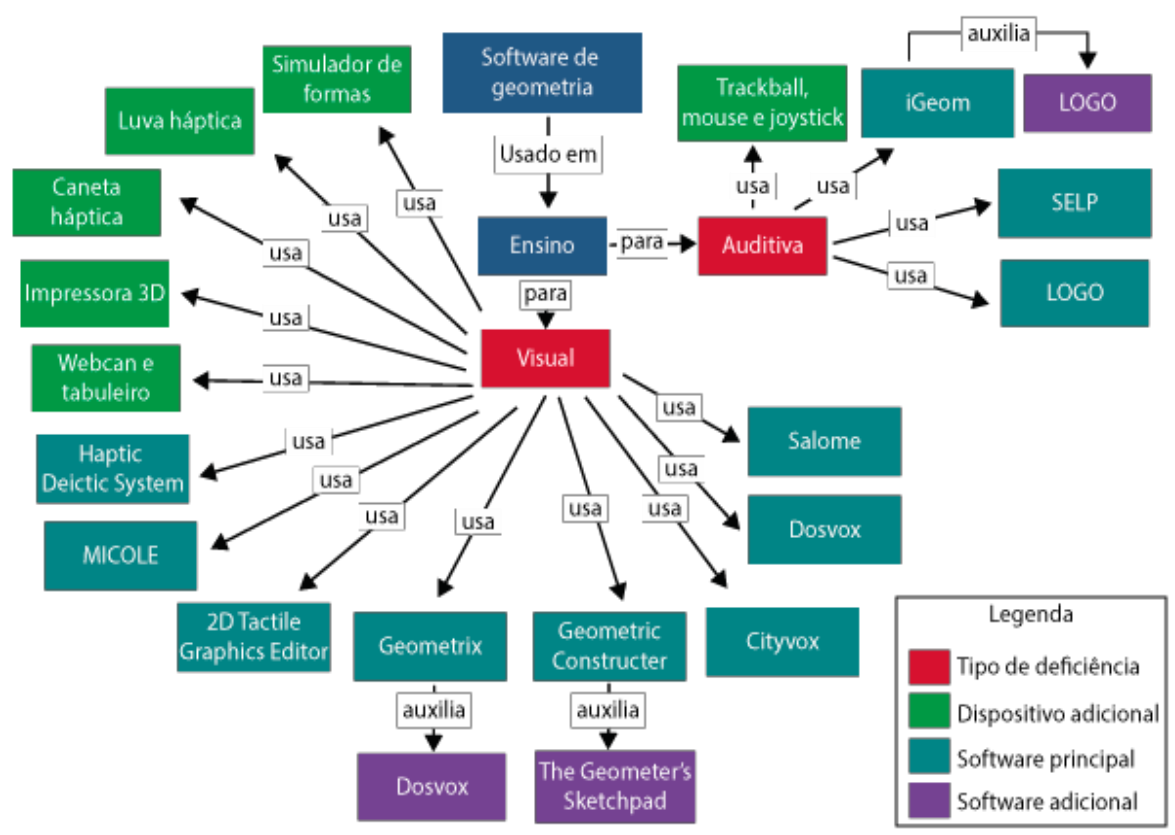

Figura 2: Mapa conceitual construído com base nos estudos primários

Por meio da Tabela 3, foi identificado os estudos primários de acordo com faixa etária dos usuário que testaram os softwares de GI, que responde a $\mathrm{QP}_{1.1}$ (Para qual faixa etária estes softwares de GI foram desenvolvidos?). A maioria dos estudos primários (12) 
não definiram uma faixa etária, correspondendo a $60 \%$. Entretanto, oito estudos definiram a faixa etária, sendo: 4 estudos voltados para crianças (20\%), 3 estudos voltados para adolescentes (15\%) e 1 estudo voltado para adultos (5\%). Com base nesses dados foi calculada a média de idade qual os estudos primários se basearam para construir o conteúdo programático e o desempenho dos participantes, e esta idade média ficou entre 10,75 anos e 32,5 anos.

Tabela 3 - Estudos primários de acordo com faixa etária.

\begin{tabular}{|c|c|c|}
\hline Categoria & Frequência & Porcentagem $(\%)$ \\
\hline Criança & 4 & $20 \%$ \\
\hline Adolescente & 3 & $15 \%$ \\
\hline Adulto & 1 & $5 \%$ \\
\hline Não definido & 12 & $60 \%$ \\
\hline
\end{tabular}

A maioria dos estudos primários investigaram a utilização de alguns dispositivos adicionais (Figura 3) para auxiliar e melhorar a experiência, correspondendo a 11 estudos (55\%). O dispositivo mais utilizado é a caneta háptica com 4 estudos $(36 \%)$ voltados para deficientes visuais, o que responde a $Q P_{2}$ (Quais dispositivos adicionais que estes softwares de GI usualmente utilizam?). Os dispositivos menos investigados foram luva háptica, simulador de formas, trackball, mouse e joystick (1 estudo cada).

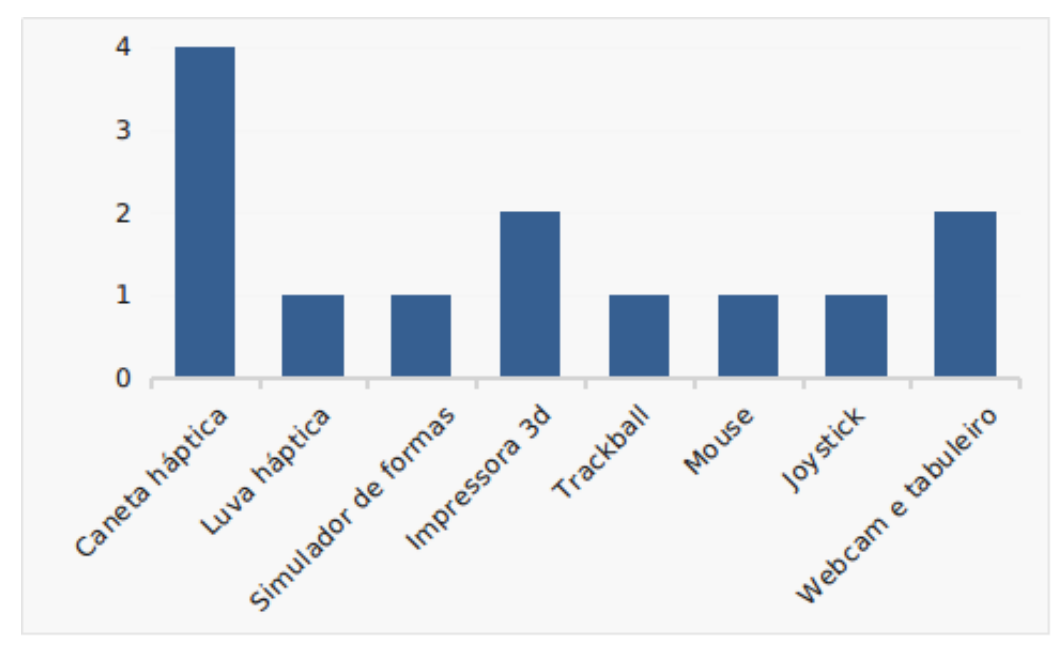

Figura 3: Dispositivos adicionais utilizados.

A fim de responder a $Q P_{5.1}$ (Quais softwares adicionais que estes softwares de GI usualmente utilizam?), identificamos softwares adicionais que podem apoiar o ensino da geometria para deficientes, além dos softwares de GI. A maioria dos estudos primários, 15 estudos correspondendo a $75 \%$, não utilizam outros softwares, como por exemplo leitor de tela. Entretanto, 5 estudos utilizaram softwares adicionais, correspondendo a $25 \%$. A Figura 4 mostra que o software mais utilizado foi o Dosvox com 2 estudos (50\%). Os demais softwares, como LOGO e The Geometer's Sketchpad, aparecem em 1 (25\%) estudo cada. 


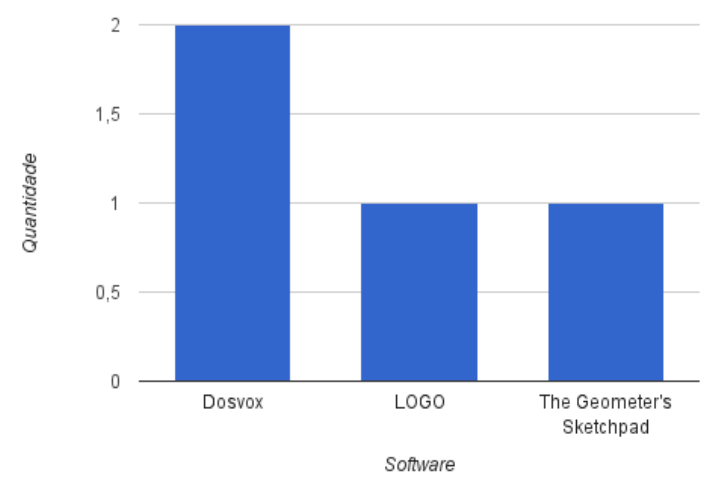

Figura 4: Dispositivos softwares utilizados.

\section{Ameaças}

Para assegurar uma seleção imparcial, as questões de pesquisa definidas e os critérios de inclusão e de exclusão dos artigos encontrados, foram definidos no início do mapeamento sistemático. No entanto, uma ameaça relacionada com a avaliação da qualidade dos estudos e artigos encontrados que foram incluídos não pode ser descartada, já que os estudos foram selecionados sem atribuir pontuações.

Outra possível ameaça identificada é a possibilidade de que alguns artigos relevantes não foram incluídos devido ao uso de um conjunto limitado de bibliotecas digitais. O sistema de classificação e as categorias elaboradas também representam uma ameaça para a validade. Como mostrado por Pretorius e Budgen (2008), a melhor maneira de separar e classificar os resultados seria após a obtenção dos artigos.

\section{Discussão e conclusão}

O principal objetivo deste mapeamento sistemático é fornecer uma visão geral do que tem sido investigado no contexto do ensino de geometria para pessoas com deficiência. Para cumprir este o objetivo, foi realizado um mapeamento sistemático. Foram definidas quatro questões a serem respondidas por esse mapeamento. $Q P_{1}$ : Quais são os principais tipos de deficiência investigados para softwares de GI? QP 1.1: Para qual faixa etária estes softwares de GI foram desenvolvidos? $\boldsymbol{Q P}_{\mathbf{2}}$ : Quais dispositivos adicionais que estes softwares de GI usualmente utilizam? $\boldsymbol{Q P}_{\mathbf{3}}$ : Quais softwares adicionais que estes softwares de GI usualmente utilizam?

De acordo com os resultados, a maioria dos estudos investigaram a deficiência visual (QP1- ver Figura 2). A faixa etária média mínima dos usuários que participaram dos testes é de 10.75 anos e a idade média máxima é 32.5 anos, onde cerca de 8 estudos (40\%) apresentaram idade dos usuários participantes e 12 estudos (60\%) não apresentaram $\left(\mathrm{QP}_{1.1}\right)$. Diversos estudos utilizaram dispositivos adicionais, cerca de 11 estudos (55\%) usaram algum dispositivo adicional e 9 (45\%) não usaram, sendo que os dispositivos usados foram caneta háptica, 4 estudos (36.37\%), e impressora 3D (QP2). Menos da metade dos estudos utilizaram algum software adicional, apenas 4 estudos (20\%), já que os que utilizaram foram 16 estudos (80\%), os softwares adicionais usados foram Dosvox, 2 estudos (50\%), Logo, 1 (20\%) e The Geometer's Sketchpad, 1 estudo (20\%) (QP3) . 
8

Esta pesquisa contribui para o avanço das tecnologias no ensino de geometria para deficientes, pois é levantado dados que mostram qual profundidade foram realizadas pesquisas sobre este assunto. Ao analisar essa pesquisa, é possível identificar que existem poucos estudos sobre o ensino de geometria para deficientes, porém pode-se perceber um pequeno crescimento no interesse deste tema atualmente. Desta forma é possível concluir: este é um campo importante para futuras pesquisas e desenvolvimento de novas tecnologias que possam melhorar o aprendizado de geometria para estudantes que tenham algum tipo de deficiência.

\section{Referências}

Arzarello, F.; Bairral, M.; Danè, C. "Moving from dragging to touchscreen: geometrical learning with geometric dynamic software." Teaching mathematics and its applications 33.1, 2014: 39-51.

Buzzi, M.; Buzzi, M.; Leporini, B.; Senette, C. "Playing with geometry: a Multimodal Android App for Blind Children." Proceedings of the 11th Biannual Conference on Italian SIGCHI Chapter. ACM, 2015.

Franciosi, Beatriz Regina Tavares. "Projeto de interfaces graficas para ensino de deficientes auditivos." 1988.

Hohenwarter, M. "Geogebra”. Disponível em: <http://www.geogebra.org/cms/pt_BR/component/contact/11-developers/1-marku s> Acesso em: 10 dez. 2016.

Gouy-Pailler, C.; Zijp-Rouzier, S.; Vidal, S.; Chêne, D. "A Haptic Based Interface to Ease Visually Impaired Pupils' Inclusion in Geometry Lessons." Universal Access in Human-Computer Interaction. Applications and Services. Springer Berlin Heidelberg, 2007. 598-606.

Ina, S. "Embodiment of 3D virtual objects for the blind by PC." ACM SIGCAPH Computers and the Physically Handicapped 60, 1998: 17-21.

Isotani, S.; Tsutsumi, M.; Brnadão, L. "O uso do computador no ensino de geometria para deficientes auditivos." Anais do III Fórum de Informática Aplicada a Pessoas Portadoras de Necessidades Especiais, 2004: 637-642.

Kiboss, J. "Effects of special e-learning program on hearing-impaired learners' achievement and perceptions of basic geometry in lower primary mathematics." Journal of Educational Computing Research 46.1, 2012: 31-59.

Kortenkamp, U.; Materlik, D. "Geometry teaching in wireless classroom environments using Java and J2ME." Science of Computer Programming, vol. 53, n.1,páginas 71-85, 2004.

Lirio, S. "A tecnologia informática como auxílio no ensino de geometria para deficientes visuais." 2006: v-115.

Martins, H. "O ensino da matemática aos deficientes auditivos." 2013: 44-f.

Moll, J.; Pysander, E. "A haptic tool for group work on geometrical concepts engaging blind and sighted pupils." ACM Transactions on Accessible Computing (TACCESS) 4.4, 2013: 14.

Oliveira, F; Cowan, H.; Fang, B.; Quek, F. "Enabling multimodal discourse for the blind." International Conference on Multimodal Interfaces and the Workshop on Machine Learning for Multimodal Interaction. ACM, 2010. 
9

Organização Mundial da Saúde. Disponível em: $<$ http://nacoesunidas.org/oms-afirma-que-existem-39-milhoes-de-cegos-no-mund o/> Acessado em: 28 mai. 2016.

Petersen, K.; Feldt, R.; Mujtaba, S.; Mattsson, . "Systematic mapping studies in software engineering." Proceedings of the 12th international conference on Evaluation and Assessment in Software Engineering, 2008: 68-77.

Pretorius, R.; Budgen, D. "A Mapping Study on Empirical Evidence Related to the Models and Forms used in the UML." In 2nd ACM-IEEE International Symposium on Empirical Software Engineering and Measurement (ESEM), 2008: 342- 344.

Rothman. "R. C.a.r. - compass and rules" Disponível em: <http://car.rene-grothmann.de/doc_en/overview.html> Acesso em: 10 dez. 2016.

Silva, L.; Broges, J.; Lima, C.; Soares, A. "Ensinando Geometria a Deficientes Visuais: o ambiente dinâmico Geometrix." Revista Brasileira de Informática na Educação 21.02, 2013: 62.

Silva, L. "Geometria e cegos - Um jogo computacional no processo de aprendizagem de trigonometria." 